\title{
SHEPHARD TYPE PROBLEMS FOR THE NEW GEOMETRIC BODY $\Gamma_{-p} K$
}

\section{WAN XIAOYAN AND WANG WEIDONG}

Abstract. Lutwak, Yang and Zhang proposed the notion of the new geometric body $\Gamma_{-p} K$. In this article, we research the Shephard-type problems for the new geometric body $\Gamma_{-p} K$.

Mathematics subject classification (2010): 52A20, 52A40. surface area.

Keywords and phrases: New geometric body, Shephard-type problem, $L_{p}$-mixed volume, $L_{p}$-affine

\section{REFERENCES}

[1] R. J. Gardner, Geometric Tomography, Second ed., Cambridge Univ. Press, Cambridge, 2006.

[2] E. Lutwak, D. Yang And G. Y. Zhang, $L_{p}$ John ellipsoids, Proc. London Math. Soc. 90 (2005), 497-520.

[3] E. Lutwak, D. Yang And G. Y. Zhang, On the $L_{p}$-Minkowski problem, Trans. Amer. Math. Soc. 356 (2003), 4359-4370.

[4] E. LUTwaK, The Brunn-Minkowski-Firey theory I: mixed volumes and the minkowski problem, J. Differential Geom. 38, 1 (1993), 131-150.

[5] E. LutwaK, The Brunn-Minkowski-Firey theory II: affine and geominimal surface areas, Adv. Math. 118, 2 (1996), 244-294.

[6] E. Lutwak And G. Y. Zhang, Blaschke-Santaló inequalities, J. Differential Geom. 47, 1 (1997), $1-16$.

[7] R. SchneIder, Convex Bodies: The Brunn-Minkowski Theory, Cambridge Univ., Press, Cambridge, 1993.

[8] J. YuAn, L. Z. ZhaO AND G. S. Leng, Inequalities for $L_{p}$-centroid body, Taiwan. J. Of Math. 11, 5 (2007), 1315-1325.

[9] L. Si, B. Xiong And W. Y. Yu, Dual $L_{p}$-affine isoperimetric inequalities, J. Inequal. Appl. 10 (2006), 1-11.

[10] W. D. Wang And G. S. Leng, $L_{p}$-mixed affine surface area, J. Math. Anal. Appl. 335, 1 (2007), 341-354.

[11] W. D. WANG AND G. S. LENG, On the $L_{p}$-version of the petty's conjectured projection inequality and applications, Taiwan. J. Of Math. 12, 5 (2008), 1-19.

[12] W. D. WANG AND G. S. LeNG, On some inequalities for the new geometric body $\Gamma_{-p} K$, Acta Math. Sin. (Chinese Series) 49, 5 (2006), 1327-1334. 